\title{
On the Nature of Rate Acceleration in the Synthesis and Fragmentation of Triazolines by Brønsted Acid: Secondary Catalysis by Water (Hydronium Triflate)
}

\author{
Supporting Information \\ Ki Bum Hong, Matthew G. Donahue, and Jeffrey N. Johnston \\ Department of Chemistry and Vanderbilt Institute of Chemical Biology, Vanderbilt University, Nashville, TN 37235-1822
}

\begin{tabular}{|c|c|}
\hline \\
\hline \\
\hline \multicolumn{2}{|c|}{ 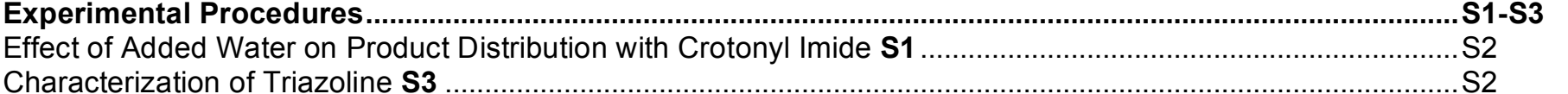 } \\
\hline \multicolumn{2}{|c|}{ 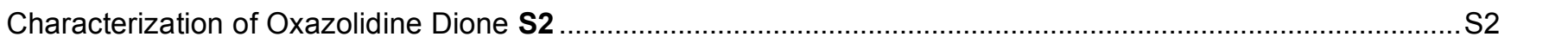 } \\
\hline \multicolumn{2}{|c|}{ (1) } \\
\hline \multicolumn{2}{|l|}{ Oxazolidine Dione 2 via Thermal Conversion ...... } \\
\hline \multicolumn{2}{|c|}{ Oxazolidine Dione 2 via Water $\left(\mathrm{H}_{2}{ }^{16} \mathrm{O}\right)$ Catalyzed Conversion } \\
\hline \multicolumn{2}{|c|}{ 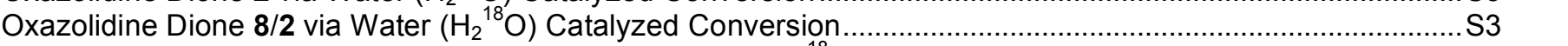 } \\
\hline \multicolumn{2}{|c|}{ Oxazolidine Dione 2 via Thermal Conversion Followed by $\mathrm{H}_{2}{ }^{18} \mathrm{O}$ Addition ........................................................ 3} \\
\hline \multicolumn{2}{|c|}{ IR Data } \\
\hline & .54 \\
\hline \multicolumn{2}{|l|}{ Triazoline } \\
\hline \multicolumn{2}{|l|}{ Oxazolidine Dione 2} \\
\hline \multicolumn{2}{|l|}{ Oxazolidine Dione 8/2. } \\
\hline \multirow{2}{*}{\multicolumn{2}{|c|}{ In Situ IR Experiments }} \\
\hline & \\
\hline Water $\left(\mathrm{H}_{2}{ }^{16} \mathrm{O}\right)$ Catalyzed & 8 \\
\hline Water $\left(\mathrm{H}_{2}{ }^{18} \mathrm{O}\right)$ Catalyze & \\
\hline & \\
\hline
\end{tabular}

\section{General Experimental Procedures}

Unless otherwise noted, all reactions were carried out under argon or nitrogen using flame or oven dried glassware and standard syringe, cannula, and septa techniques, when necessary. Trifluoromethanesulfonic acid $(10 \mathrm{~mL})$ was distilled under reduced pressure $(5 \mathrm{mmHg})$ from trifluoromethanesulfonic anhydride $(1 \mathrm{~mL})$ from $70-80{ }^{\circ} \mathrm{C}$ and stored in a dessicator. Acetonitrile $\left(\mathrm{CH}_{3} \mathrm{CN}\right)$, tetrahydrofuran $(\mathrm{THF})$, and dichloromethane $\left(\mathrm{CH}_{2} \mathrm{Cl}_{2}\right)$ were dried by passage through a column of activated alumina as described by Grubbs. ${ }^{1}$ Triethylamine was distilled from calcium hydride under argon. $\mathrm{H}_{2}{ }^{18} \mathrm{O}$ (95\% atom product of Isotec) was purchased from Aldrich. Flash column chromatography was performed using Sorbent Technologies 40-63 $\mathrm{m}$, pore size $60 \AA$ silica gel with solvent systems indicated. Analytical thin layer chromatography was performed using Sorbent Technologies $250 \mu \mathrm{m}$ glass-backed UV254 silica gel plates that were visualized by fluorescence upon $254 \mathrm{~nm}$ irradiation then by staining upon heating with phosphomolybdic acid or potassium iodoplatinate. Solvent removal was effected by rotary evaporation under water aspirator vacuum $(\sim 25-40$ $\mathrm{mmHg}$ ).

All melting points were taken with a Laboratory Devices Mel-Temp melting point apparatus and are uncorrected. Proton nuclear magnetic resonance spectra were recorded on Bruker DPX-400 or Bruker DRX-500 spectrometers and are recorded in parts per million from internal chloroform on the $\delta$ scale and are reported as follows: chemical shift [multiplicity ( $\mathrm{s}=$ singlet, $\mathrm{d}=$ doublet, $\mathrm{t}=$ triplet, $\mathrm{q}=$ quartet, $q u=q u i n t e t, \mathrm{~m}=$ multiplet), coupling constant(s) in hertz, integration]. ${ }^{3} \mathrm{C}$ NMR data were recorded on either a Bruker DPX-400 or Bruker DRX-500 spectrometer.

A Mettler-Toledo ReactIR iC-10 equipped with a K-6 conduit immersible probe with SiComp ATR probe tip connected to a liquid nitrogen cooled MCT detector was used for in situ reaction monitoring at 8 wavenumber resolution. The instrument was outfitted with a Dell Latitude D505 laptop running iC IR version 2.0. Industrial grade nitrogen was used to continually purge the system. The external temperature of the isopropanol bath was maintained at $-20{ }^{\circ} \mathrm{C}$ using a Thermo Electron Corporation NESLAB CC 100 Immersion Cooler with the controller set to $-25^{\circ} \mathrm{C}$. The acetonitrile was subtracted from the reaction and all 3D spectra were baseline corrected with baseline offset at $1985 \mathrm{~cm}^{-1}$. The raw data was exported into Microsoft Excel to produce the IR spectra. The trendlines for the $1830 \mathrm{~cm}^{-1}$ and $1711 \mathrm{~cm}^{-1}$ peaks were formatted to height to single point at $1985 \mathrm{~cm}^{-1}$. The data corresponding to the conversion time period of 1 hour for each experiment was chosen and the time was then converted into minutes and subsequently into a relative time period.

Mass spectra were recorded on a Kratos MS-80 spectrometer by use of chemical ionization $(\mathrm{Cl})$.

\footnotetext{
${ }^{1}$ Pangborn, A. B.; Giardello, M.A.; Grubbs, R. H.; Rosen, R. K.; Timmers, F. J. Organometallics 1996, 15, 1518-1520.
} 
Table S1. Effect of Added Water on the Product Distribution of Triflic Acid Promoted Azide-Olefin Additions ${ }^{a}$

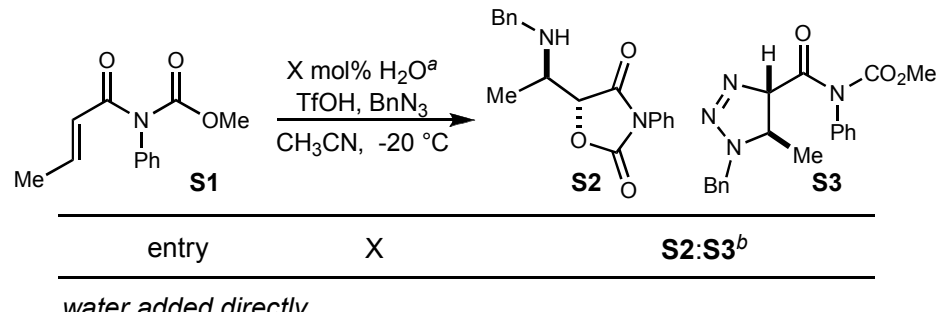

\begin{tabular}{ccc} 
water added directly & & \\
$1^{c}$ & 0 & $95: 5$ \\
2 & 0 & $5: 95$ \\
3 & 10 & $33: 67$ \\
4 & 100 & $78: 22$ \\
5 & 200 & $95: 5$ \\
\hdashline water premixed with $T f O H$ & \\
6 & 10 & $50: 50$ \\
7 & 50 & $95: 5$ \\
8 & 100 & $95: 5$ \\
9 & 1000 & $-d$ \\
\hline
\end{tabular}

a General conditions: $\mathrm{BnN}_{3}$ (1.5 equiv.) and the olefin (1 equiv) in $\mathrm{CH}_{3} \mathrm{CN}$ $(0.33 \mathrm{M})$ were treated with triflic acid at $-20^{\circ} \mathrm{C}$. The triflic acid was first premixed with the indicated amount of water to create a stock solution. For additional procedural variations, resulting in similar observations, see the Supporting Information. ${ }^{b}$ Measured by ${ }^{1} \mathrm{H}$ NMR analysis of the crude reaction mixture after reaction quench $\left(\mathrm{Et}_{3} \mathrm{~N}, 2\right.$ equiv.) at the reaction temperature and removal of volatiles by high vacuum. ${ }^{\circ}$ Reaction mixture was warmed to room temperature prior to quench. ${ }^{d}$ Only hydrolysis of imide observed.

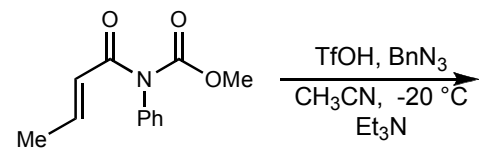

$(63 \%)$

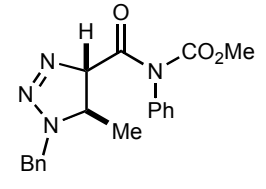

Characterization of Triazoline (S3): To a solution of acetonitrile $(0.3 \mathrm{~mL})$ and imide (S1) $(20 \mathrm{mg}, 0.09 \mathrm{mmol})$ cooled to $-20{ }^{\circ} \mathrm{C}$ was added triflic acid $(24 \mu \mathrm{L}, 0.13 \mathrm{mmol})$. After $10 \mathrm{~min}$, benzyl azide (16 $\mu \mathrm{L}, 0.18 \mathrm{mmol}$ ) was added. The reaction mixture was quenched after $60 \mathrm{~min}$ with triethylamine $(25 \mu \mathrm{L}, 0.13 \mathrm{mmol})$. The solvent was removed in vacuo, and the residue was chromatographed to afford

triazoline (S3) as a colorless oil (20 mg, 63\%). $R_{f}=0.50$ (30\% EtOAc/hexanes); IR (film) 3063, 3031, 2955, 2086, 1747, 1703, 1683, $1652 \mathrm{~cm}^{-1} ;{ }^{1} \mathrm{H}$ NMR $\left(400 \mathrm{MHz}, \mathrm{CDCl}_{3}\right) \delta 7.07-7.38(\mathrm{~m}, 10 \mathrm{H}), 5.72(\mathrm{~d}, J=11.2 \mathrm{~Hz}, 1 \mathrm{H}), 4.90(\mathrm{~d}, J=15.2 \mathrm{~Hz}$, $1 \mathrm{H}), 4.63(\mathrm{~d}, J=15.2 \mathrm{~Hz}, 1 \mathrm{H}), 3.76-3.79(\mathrm{~m}, 1 \mathrm{H}), 3.74(\mathrm{~s}, 3 \mathrm{H}), 1.14(\mathrm{~d}, J=6.4 \mathrm{~Hz}, 3 \mathrm{H}) ;{ }^{13} \mathrm{C} \mathrm{NMR}\left(100 \mathrm{MHz}, \mathrm{CDCl}_{3}\right) \mathrm{ppm}$ 169.8, 154.2, 137.4, 135.4, 129.0, 128.4, 128.2, 128.0, 127.8, 127.6, 85.7, 54.3, 54.0, 51.9, 16.7; HRMS (Cl): Exact mass calcd for $\mathrm{C}_{19} \mathrm{H}_{20} \mathrm{~N}_{4} \mathrm{O}_{3}[\mathrm{M}]^{+}$352.1530. Found 352.1530.

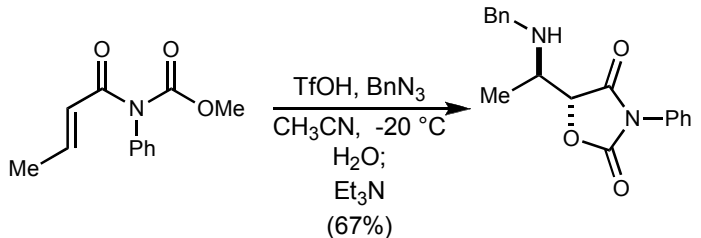

$(67 \%)$
Characterization of Oxazolidine Dione (S2): To a solution of acetonitrile $(5.0 \mathrm{~mL})$ and imide $(\mathbf{S} 1)(109 \mathrm{mg}, 0.50 \mathrm{mmol})$ cooled to $-20^{\circ} \mathrm{C}$ was added triflic acid $(88 \mu \mathrm{L}, 1.00 \mathrm{mmol})$. After $10 \mathrm{~min}$, benzyl azide $(99 \mu \mathrm{L}, 0.75$ $\mathrm{mmol}$ ) was added. After $60 \mathrm{~min}$, water (distilled, deionized $9 \mu \mathrm{L}, 0.5 \mathrm{mmol}$ ) was added and stirring continued cold for $24 \mathrm{~h}$. The reaction mixture was quenched with triethylamine $(139 \mu \mathrm{L}, 1.00 \mathrm{mmol})$. The solvent was removed in vacuo, and the residue was chromatographed to afford oxazolidine dione $\mathbf{S} 2$ as a colorless oil $(103 \mathrm{mg}, 67 \%)$. $\mathrm{R}_{f}=0.30$ (30\% EtOAc/hexanes); IR (film) $3298,1752,1683 \mathrm{~cm}^{-1}$; ${ }^{1} \mathrm{H}$ NMR $\left(400 \mathrm{MHz}, \mathrm{CDCl}_{3}\right) \delta 7.19-7.37(\mathrm{~m}, 10 \mathrm{H}), 4.96(\mathrm{~d}, J=3.2 \mathrm{~Hz}, 1 \mathrm{H}), 3.93(\mathrm{~d}, J=13.2 \mathrm{~Hz}, 1 \mathrm{H}), 3.88(\mathrm{~d}, J=13.2 \mathrm{~Hz}$, $1 \mathrm{H}), 3.39(\mathrm{dq}, J=6.8,3.2 \mathrm{~Hz}, 1 \mathrm{H}), 1.30(\mathrm{~d}, J=6.8 \mathrm{~Hz}, 3 \mathrm{H})$, the $\mathrm{NH}$ was not recorded; ${ }^{13} \mathrm{C} \mathrm{NMR}\left(100 \mathrm{MHz}, \mathrm{CDCl}_{3}\right) \mathrm{ppm}$ 170.7, 154.3, 139.5, 130.7, 129.2, 128.8, 128.4, 127.9, 127.1, 125.6, 81.6, 53.5, 51.7, 15.0; HRMS (Cl): Exact mass calcd for $\mathrm{C}_{18} \mathrm{H}_{18} \mathrm{~N}_{2} \mathrm{O}_{3}[\mathrm{M}+\mathrm{H}]^{+}$311.1390. Found 311.1394.<smiles>C=CC(=O)N(Cc1ccccc1)C(=O)OC</smiles>

$(54 \%)$<smiles>CC(=O)N(Cc1ccccc1)C(=O)C1CN(Cc2ccccc2)N=N1</smiles>

Characterization of Triazoline (3): To acetonitrile $(8.0 \mathrm{~mL})$ in the reaction vessel cooled to $-20{ }^{\circ} \mathrm{C}$ was added imide $1(850 \mu \mathrm{L}, 4.56 \mathrm{mmol})$, followed by triflic acid $(800 \mu \mathrm{L}, 9.12 \mathrm{mmol})$ via syringe and stirring continued for $75 \mathrm{~min}$. To the solution was added benzyl azide $(610 \mu \mathrm{L}$, 
$4.56 \mathrm{mmol})$ of via syringe. After $40 \mathrm{~min}$, the reaction mixture was quenched with triethylamine $(1.40 \mathrm{~mL}, 4.56 \mathrm{mmol}) \mathrm{via}$ syringe. The solvent was removed in vacuo. The residue was purified by chromatography (hexanes-ethyl acetate, $10: 1 \rightarrow 5: 1 \rightarrow 2: 1)$ using a jacketed column circulating ice-water to give triazoline 3 as a faint yellow oil that solidified after being triturated with $1 \mathrm{~mL}$ of hexanes-ethyl acetate $(10: 1)(868 \mathrm{mg}, 54 \%): \mathrm{R}_{f}=0.14$ (hexanes-ethyl acetate, $5: 1$; uv; potassium iodoplatinate stained brown); $\mathrm{mp} 81-84{ }^{\circ} \mathrm{C}$; IR (neat) $1740,1704 \mathrm{~cm}^{-1} ;{ }^{1} \mathrm{H}$ NMR $\left(400 \mathrm{MHz}, \mathrm{CDCl}_{3}\right) \delta 7.25-7.35$ $(\mathrm{m}, 10 \mathrm{H}), 6.09(\mathrm{dd}, J=12.4,10.8 \mathrm{~Hz}, 1 \mathrm{H}), 4.99(\mathrm{~d}, J=15.2 \mathrm{~Hz}, 1 \mathrm{H}), 4.97(\mathrm{~d}, J=14.8 \mathrm{~Hz}, 1 \mathrm{H}), 4.93(\mathrm{~d}, J=14.8 \mathrm{~Hz}, 1 \mathrm{H})$, $4.68(\mathrm{~d}, J=15.2 \mathrm{~Hz}, 1 \mathrm{H}), 3.84(\mathrm{~s}, 3 \mathrm{H}), 3.37(\mathrm{dd}, J=10.2,10.2 \mathrm{~Hz}, 1 \mathrm{H}), 3.25(\mathrm{dd}, J=12.4,10.0 \mathrm{~Hz}, 1 \mathrm{H}) ;{ }^{13} \mathrm{C}$ NMR $(100$ $\mathrm{MHz}, \mathrm{CDCl}_{3}$ ) ppm 170.2, 154.8, 136.9, 135.5, 128.7, 128.5, 128.2, 127.9, 127.6, 127.4, 80.0, 54.4, 54.1, 48.0, 46.8.

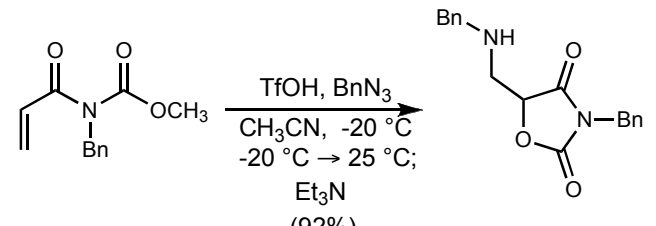

$(92 \%)$

Oxazolidine Dione (2) via Thermal Conversion: To acetonitrile $(2.0 \mathrm{~mL})$ in the reaction vessel cooled to $-20^{\circ} \mathrm{C}$ was added imide $1(149 \mu \mathrm{L}, 0.80 \mathrm{mmol})$, followed by triflic acid $(141 \mu \mathrm{L}, 1.60 \mathrm{mmol})$. After $25 \mathrm{~min}$, benzyl azide (106 $\mu \mathrm{L}, 0.80 \mathrm{mmol}$ ) was added via syringe. After $40 \mathrm{~min}$, the $-20{ }^{\circ} \mathrm{C}$ bath was replaced with a $23^{\circ} \mathrm{C}$ bath. After $80 \mathrm{~min}$, the reaction mixture was quenched with triethylamine $(223 \mu \mathrm{L}, 1.60 \mathrm{mmol})$ via syringe. The solvent was removed in vacuo, and the residue was chromatographed (hexanes-ethyl acetate, $10: 1 \rightarrow 5: 1 \rightarrow 2: 1)$ to afford oxazolidine dione 2 as a yellow oil $(229 \mathrm{mg}, 92 \%)$. Upon standing at rt, repeated scratching with a glass rod caused the oil to solidify then was triturated with $0.5 \mathrm{~mL}$ diethyl ether-methanol (10:1) to give oxazolidine dione 2 as a white solid: $\mathrm{R}_{f}=0.41$ (hexanes-ethyl acetate, 2:1; uv; phosphomolybdic acid stained dark blue); $\mathrm{mp} 74-76{ }^{\circ} \mathrm{C}$; IR (neat oil) 1818, $1737 \mathrm{~cm}^{-1}$; ${ }^{1} \mathrm{H}$ NMR $\left(500 \mathrm{MHz}, \mathrm{CDCl}_{3}\right) \delta$ 7.37-7.45 (m, 2H), 7.29-7.35 (m, 4H), $7.26(\mathrm{~d}, J=7.0 \mathrm{~Hz}, 2 \mathrm{H})$, $7.19(\mathrm{~d}, J=7.0 \mathrm{~Hz}, 2 \mathrm{H}), 4.82(\mathrm{dd}, J=3.7,3.7 \mathrm{~Hz}, 1 \mathrm{H}), 4.73(\mathrm{~d}, J=14.5 \mathrm{~Hz}, 1 \mathrm{H}), 4.67(\mathrm{~d}, J=14.5 \mathrm{~Hz}, 1 \mathrm{H}), 3.77(\mathrm{~d}, J=2.5$ $\mathrm{Hz}, 2 \mathrm{H}$ ), $3.21(\mathrm{dd}, J=13.5,3.5 \mathrm{~Hz}, 1 \mathrm{H}), 3.10(\mathrm{dd}, J=13.7,3.7 \mathrm{~Hz}, 1 \mathrm{H}), 1.51(\mathrm{br} \mathrm{s}, 1 \mathrm{H}) ;{ }^{13} \mathrm{C} \mathrm{NMR}\left(125 \mathrm{MHz}, \mathrm{CDCl}_{3}\right) \mathrm{ppm}$ 172.0, 155.4, 139.3, 134.6, 128.8, 128.7, 128.4, 128.2, 127.9, 127.1, 80.1, 53.8, 47.7, 43.6; HRMS (Cl): Exact mass calculated for $\mathrm{C}_{18} \mathrm{H}_{18} \mathrm{~N}_{2} \mathrm{O}_{3}[\mathrm{M}+\mathrm{H}]^{+} 311.1385$, found 311.1390 .

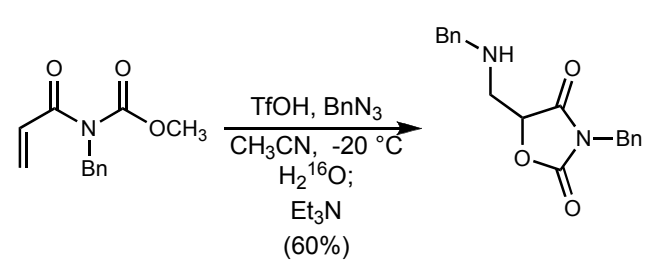

Oxazolidine Dione (2) via Water $\left(\mathrm{H}_{2}{ }^{16} \mathrm{O}\right)$ Catalyzed Conversion: To acetonitrile $(2.0 \mathrm{~mL})$ in the reaction vessel cooled to $-20^{\circ} \mathrm{C}$ was added imide $1(149 \mu \mathrm{L}, 0.80 \mathrm{mmol})$, followed by triflic acid $(141 \mu \mathrm{L}, 1.60 \mathrm{mmol})$ via syringe and stirring continued for $30 \mathrm{~min}$. To the solution was added benzyl azide (106 $\mu \mathrm{L}, 0.80 \mathrm{mmol}$ ) via syringe. After $75 \mathrm{~min}$, water (distilled, deionized, 14 $\mu \mathrm{L}, 0.80 \mathrm{mmol}$ ) was added via syringe and stirring continued for $5 \mathrm{~h}$. The reaction mixture was quenched with triethylamine $(223 \mu \mathrm{L}, 1.60 \mathrm{mmol})$ via syringe. The solvent was removed in vacuo, and the residue was chromatographed (hexanes-ethyl acetate, $10: 1 \rightarrow 5: 1 \rightarrow 2: 1$ ) furnishing oxazolidine dione 2 with spectral properties identical to those above (150 $\mathrm{mg}, 60 \%$ ).

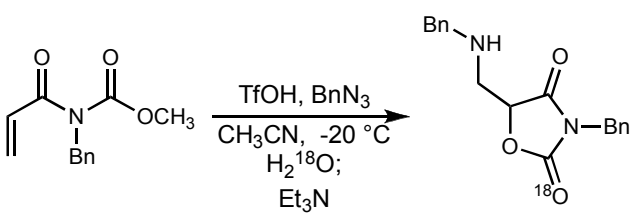

$(78 \%)$

Oxazolidine Dione (8) via Water $\left(\mathrm{H}_{2}{ }^{18} \mathrm{O}\right)$ Catalyzed Conversion: To acetonitrile $(8.0 \mathrm{~mL})$ in the reaction vessel cooled to $-20^{\circ} \mathrm{C}$ was added imide 1 ( $850 \mu \mathrm{L}, 4.56 \mathrm{mmol})$ followed by triflic acid $(800 \mu \mathrm{L}, 9.12 \mathrm{mmol})$ via syringe and stirring continued for $20 \mathrm{~min}$. To the solution was added benzyl azide (610 $\mu \mathrm{L}, 4.56 \mathrm{mmol})$ via syringe. After $3 \mathrm{~h}, \mathrm{H}_{2}{ }^{18} \mathrm{O}(95 \%$ atom, $91 \mu \mathrm{L}, 4.56 \mathrm{mmol})$ was added via syringe. After $2.3 \mathrm{~h}$, the reaction mixture was quenched with triethylamine $(1.40 \mathrm{~mL}, 4.56 \mathrm{mmol})$ of via syringe. The solvent was removed in vacuo, and the residue was chromatographed (hexanes-ethyl acetate, 10:1 $\rightarrow 5: 1 \rightarrow 2: 1$ ) to afford oxazolidine dione 8 as a light yellow oil that was scratched repeatedly with a glass pipet and subsequently solidified $(1.11 \mathrm{~g}, 78 \%): R_{f} 0.41$ (hexanes-ethyl acetate, 2:1; uv; phosphomolybdic acid stained dark blue); mp 75-76 ${ }^{\circ} \mathrm{C}$; IR (neat) $1818\left(\mathrm{C}={ }^{16} \mathrm{O}\right), 1799$ $\left(\mathrm{C}={ }^{18} \mathrm{O}\right), 1723 \mathrm{~cm}^{-1} ;{ }^{1} \mathrm{H}$ NMR $\left(500 \mathrm{MHz}, \mathrm{CDCl}_{3},\right) \delta$ 7.37-7.45 (m, 2H), 7.27-7.36 (m, 4H), 7.25 (d, $\left.J=7.0 \mathrm{~Hz}, 2 \mathrm{H}\right), 7.19(\mathrm{~d}$, $J=7.0 \mathrm{~Hz}, 2 \mathrm{H}), 4.82(\mathrm{dd}, J=3.5,3.5 \mathrm{~Hz}, 1 \mathrm{H}), 4.73(\mathrm{~d}, J=14.5 \mathrm{~Hz}, 1 \mathrm{H}), 4.67(\mathrm{~d}, J=14.5 \mathrm{~Hz}, 1 \mathrm{H}), 3.77(\mathrm{~d}, J=2.5 \mathrm{~Hz}$, $2 \mathrm{H}), 3.21(\mathrm{dd}, J=13.5,3.5 \mathrm{~Hz}, 1 \mathrm{H}), 3.11(\mathrm{dd}, J=14.0,4.0 \mathrm{~Hz}, 1 \mathrm{H}), 1.50(\mathrm{br} \mathrm{s}, 1 \mathrm{H}) ;{ }^{13} \mathrm{C} \mathrm{NMR}\left(125 \mathrm{MHz} \mathrm{CDCl}_{3}\right)$ ppm 172.0, $155.43\left({ }^{13} \mathrm{C}={ }^{16} \mathrm{O}\right), 155.40\left({ }^{13} \mathrm{C}={ }^{18} \mathrm{O}\right), 139.3,134.6,128.7,128.44,128.42,128.2,127.9,127.1,80.1,53.8$, 47.7, 43.6; HRMS (Cl): exact mass calculated for $\mathrm{C}_{18} \mathrm{H}_{18} \mathrm{~N}_{2}{ }^{16} \mathrm{O}_{3}[\mathrm{M}+\mathrm{H}]^{+}$311.1385. Found 311.1390. HRMS (Cl): exact mass calculated for $\mathrm{C}_{18} \mathrm{H}_{18} \mathrm{~N}_{2}{ }^{16} \mathrm{O}_{2}{ }^{18} \mathrm{O}_{1}[\mathrm{M}+\mathrm{H}]^{+} 313.1443$. Found 313.1433 . The ratio of $\mathrm{C}_{18} \mathrm{H}_{18} \mathrm{~N}_{2}{ }^{16} \mathrm{O}_{3}: \mathrm{C}_{18} \mathrm{H}_{18} \mathrm{~N}_{2}{ }^{16} \mathrm{O}_{2}{ }^{18} \mathrm{O}_{1}$ was $3: 5$. 

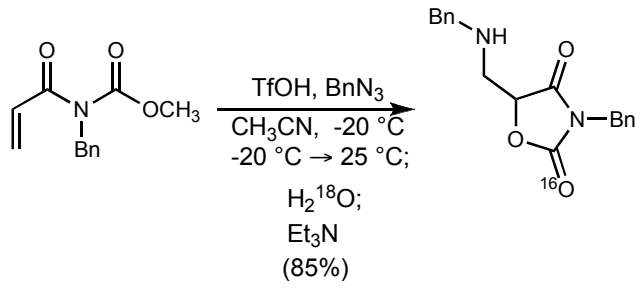

$(8 \%)$

Oxazolidine Dione (2) via Thermal Conversion Followed by $\mathrm{H}_{2}{ }^{18} \mathrm{O}$ Addition: To acetonitrile $(8.0 \mathrm{~mL})$ in the reaction vessel cooled to $-20{ }^{\circ} \mathrm{C}$ was added imide $1(850 \mu \mathrm{L}, 4.56 \mathrm{mmol})$ followed by triflic acid $(800 \mu \mathrm{L}$, $9.12 \mathrm{mmol}$ ) via syringe and stirring continued for $10 \mathrm{~min}$. To the solution was added benzyl azide $(610 \mu \mathrm{L}, 4.56 \mathrm{mmol})$ via syringe. After $2 \mathrm{~h}$, the -20 ${ }^{\circ} \mathrm{C}$ bath was replaced with a $23{ }^{\circ} \mathrm{C}$ bath. After $8 \mathrm{~h}, \mathrm{H}_{2}{ }^{18} \mathrm{O}(95 \%$ atom; $91 \mu \mathrm{L}$, $4.56 \mathrm{mmol}$ ) was added via syringe and stirring continued for $12 \mathrm{~h}$. The reaction mixture was quenched with triethylamine $(1.40 \mathrm{~mL}, 4.56 \mathrm{mmol})$ via syringe. The solvent was removed in vacuo and the residue was chromatographed (hexanes-ethyl acetate, $10: 1 \rightarrow 5: 1 \rightarrow 2: 1)$ to provide oxazolidine dione 2 with spectral properties identical to $\mathrm{C}_{18} \mathrm{H}_{18} \mathrm{~N}_{2}{ }^{16} \mathrm{O}_{3}(1.21 \mathrm{~g}, 85 \%)$. Mass spectrometry recorded no $\mathrm{m} / \mathrm{z}$ peak at $\mathrm{C}_{18} \mathrm{H}_{18} \mathrm{~N}_{2}{ }^{16} \mathrm{O}_{2}{ }^{18} \mathrm{O}_{1}[\mathrm{M}+\mathrm{H}]^{+} 313.1443$.

\section{IR Spectra}

Imide 1

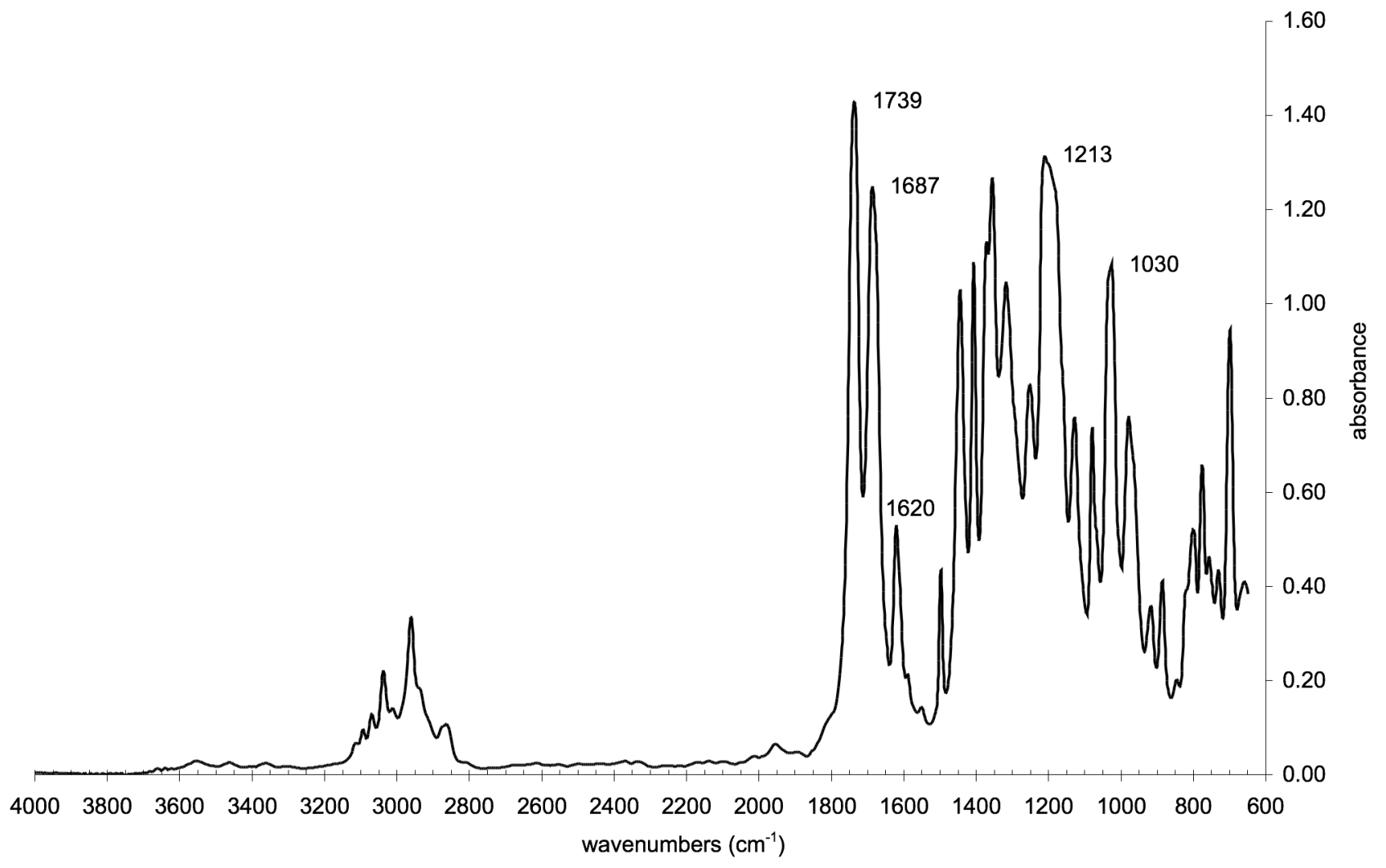


Triazoline 3
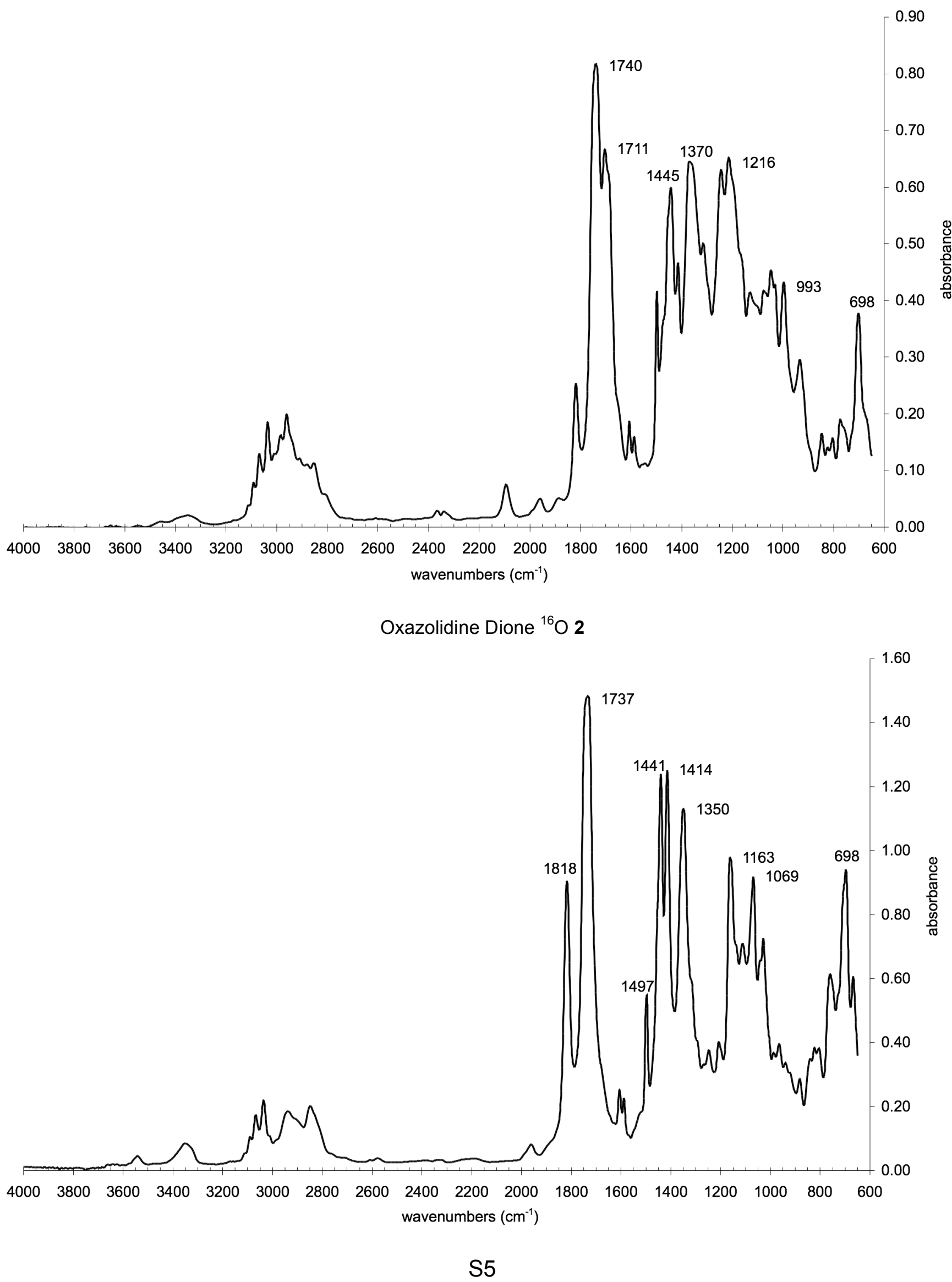


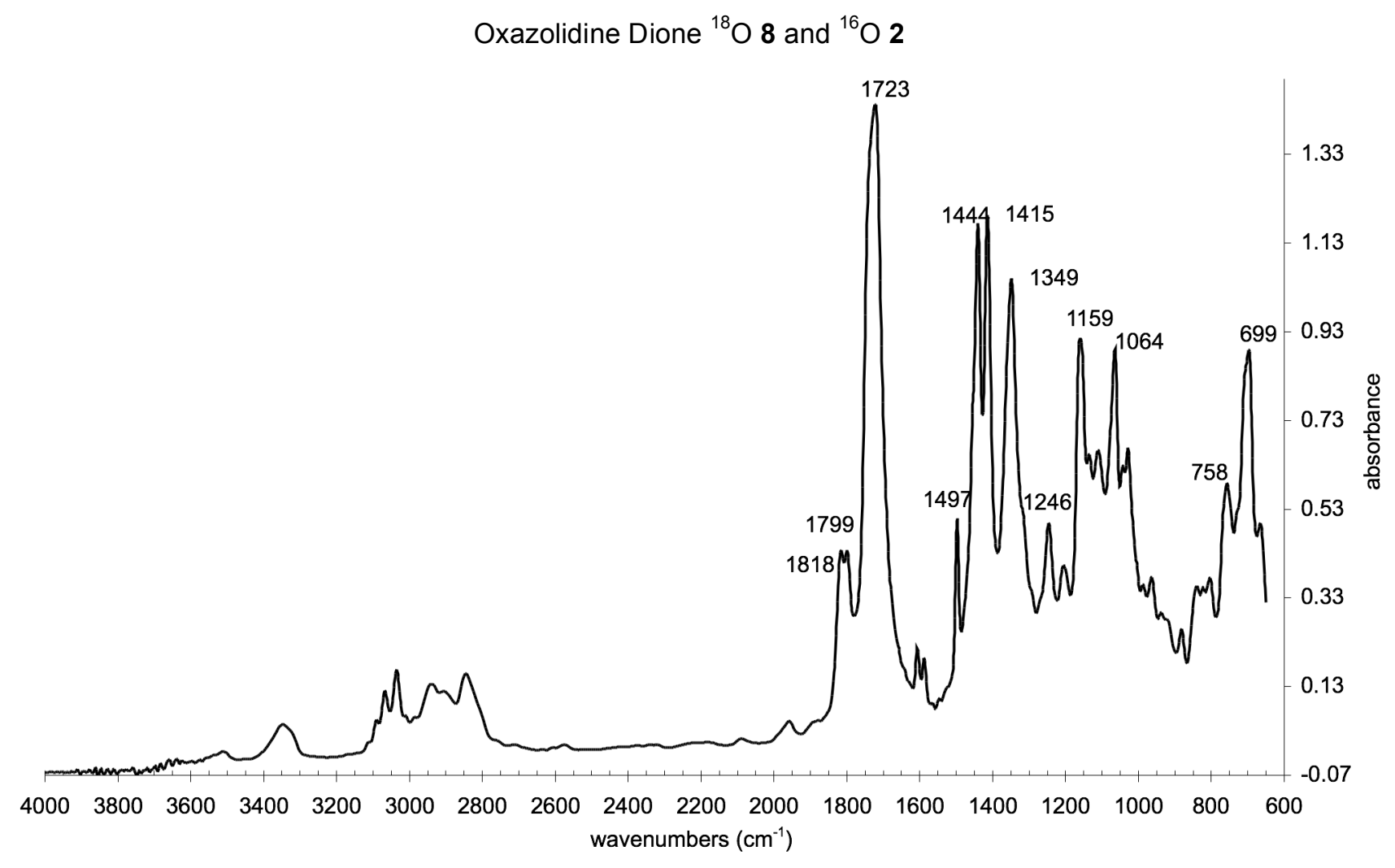

Expansion of $1850-1650 \mathrm{~cm}^{-1}$ Region Oxazolidine Dione ${ }^{18} \mathrm{O} 8$ and ${ }^{16} \mathrm{O} 2$

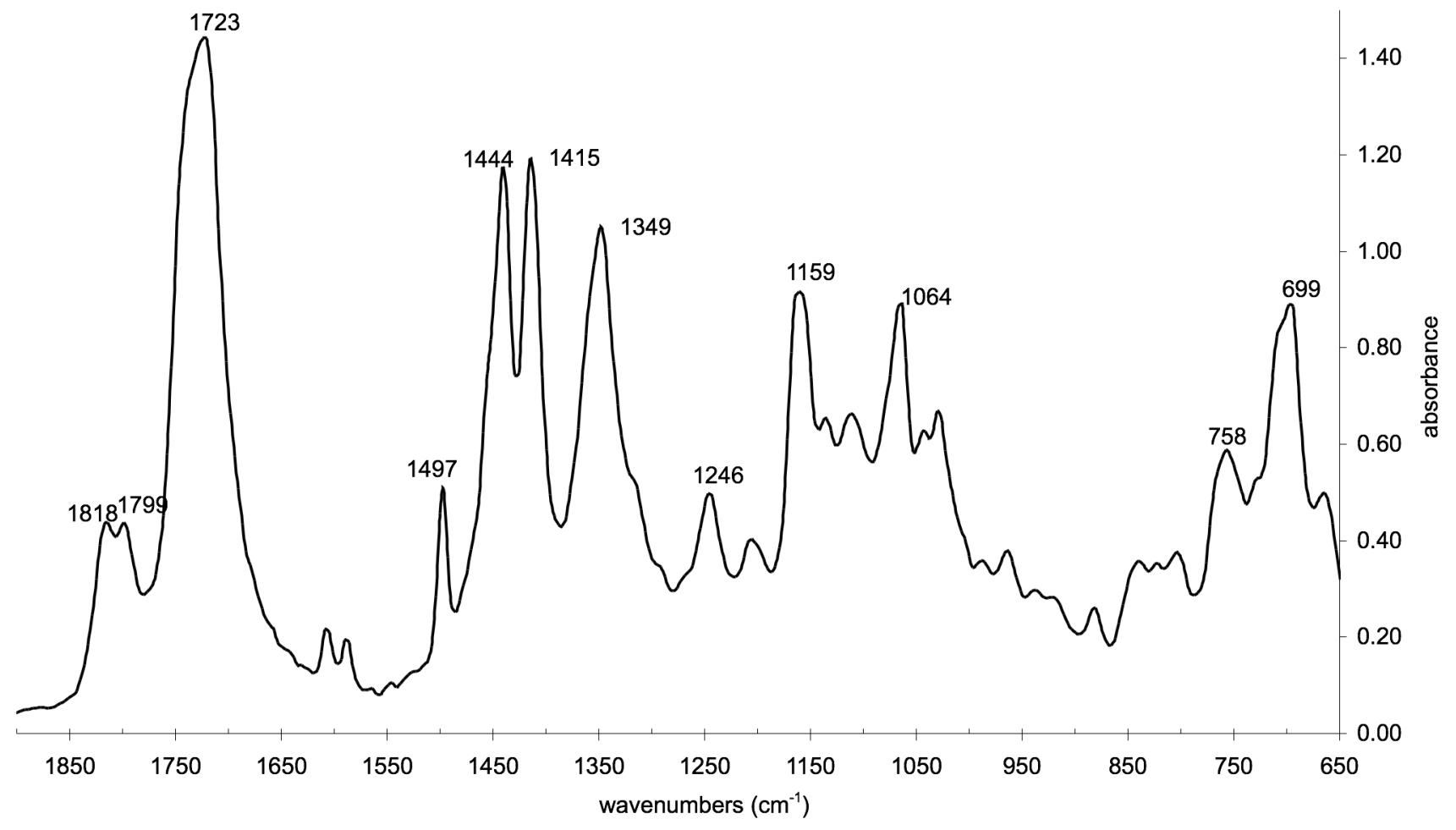




\section{In Situ ReactIR Experiments}

Thermal Conversion $1850-1650 \mathrm{~cm}^{-1}$
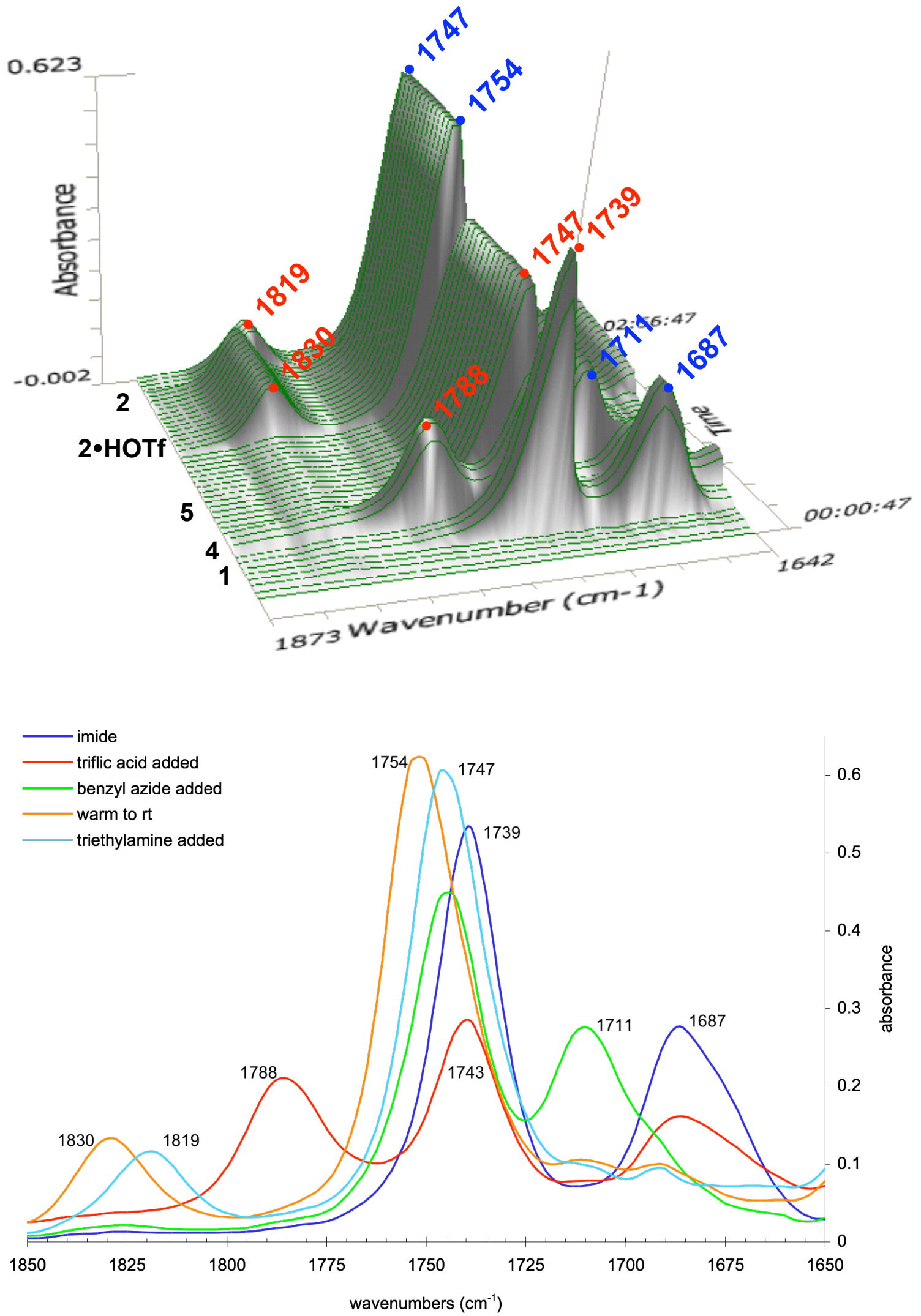
Water $\left(\mathrm{H}_{2}{ }^{16} \mathrm{O}\right)$ Catalyzed Conversion $1850-1650 \mathrm{~cm}^{-1}$
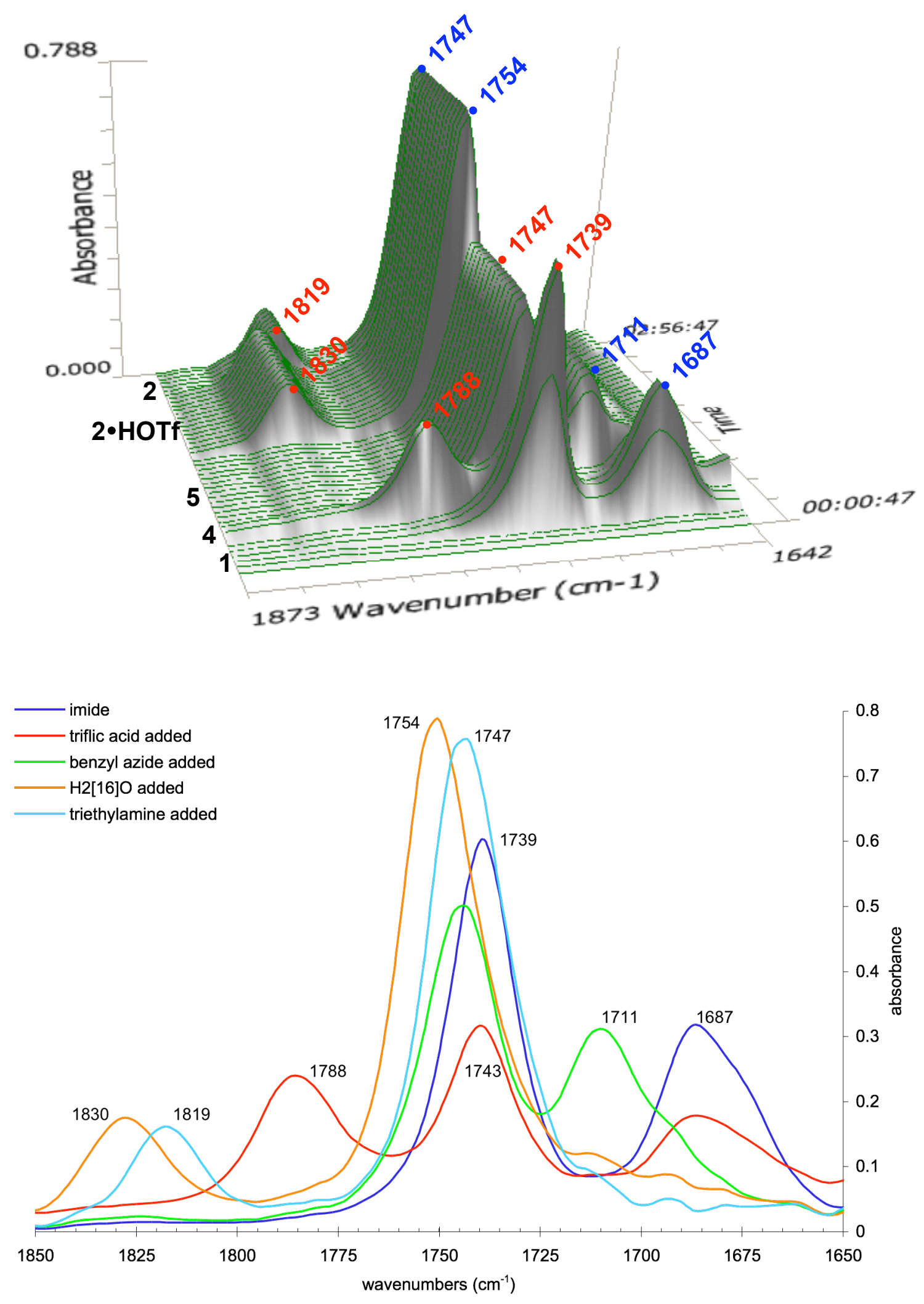
$\mathrm{H}_{2}{ }^{18} \mathrm{O}$ Catalyzed Conversion of Triazoline to Oxazolidine Dione $1850-1650 \mathrm{~cm}^{-1}$
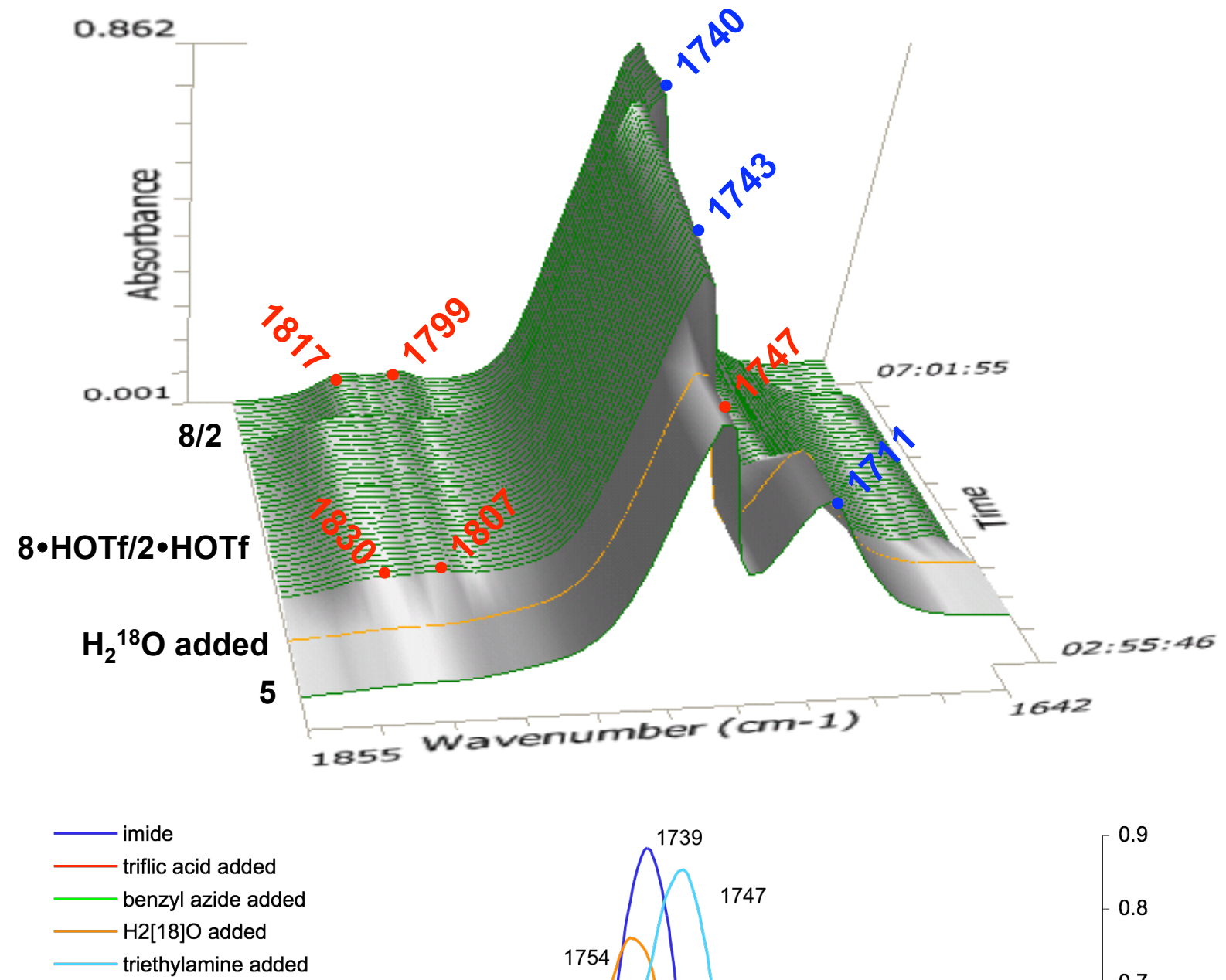

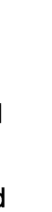

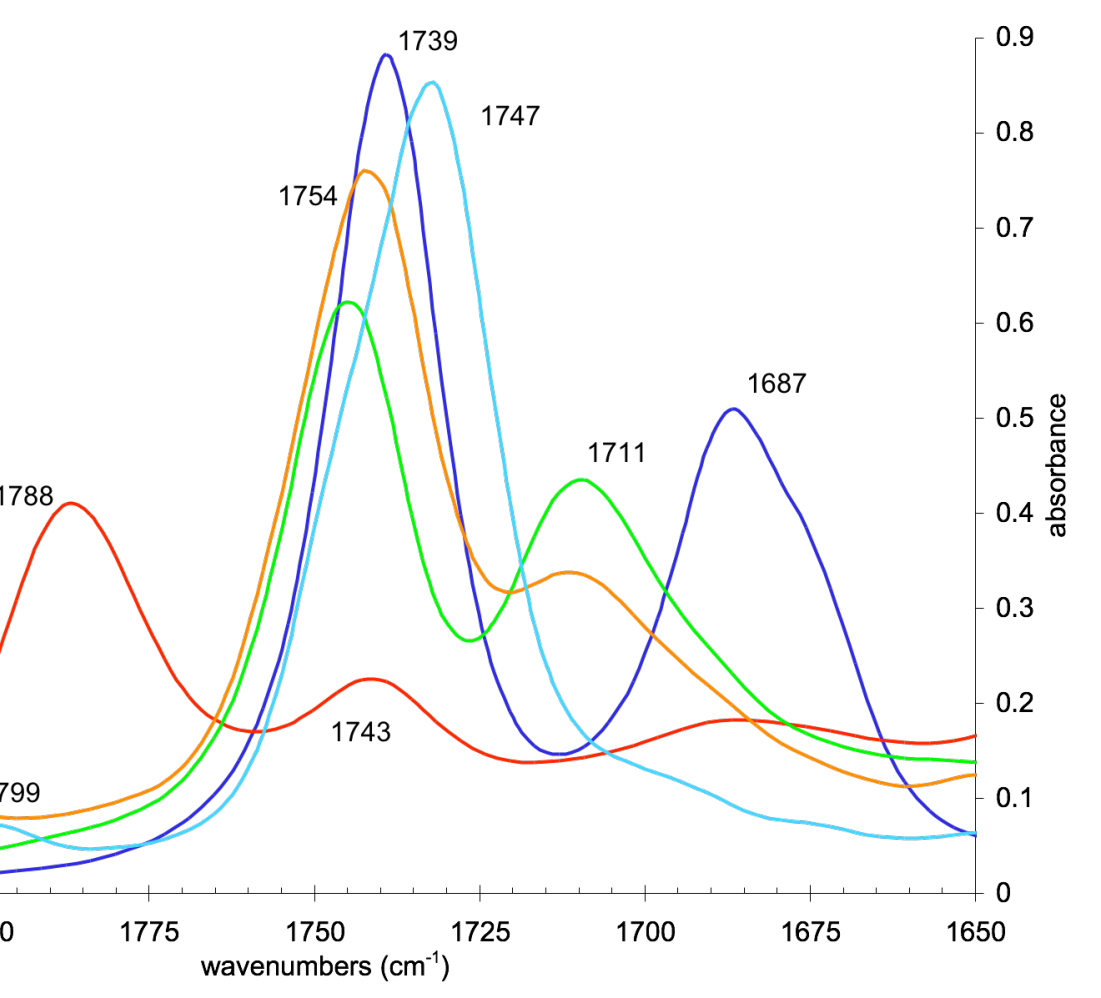


$\mathrm{H}_{2}{ }^{18} \mathrm{O}$ Catalyzed Conversion of Triazoline to Oxazolidine Dione $1850-1775 \mathrm{~cm}^{-1}$

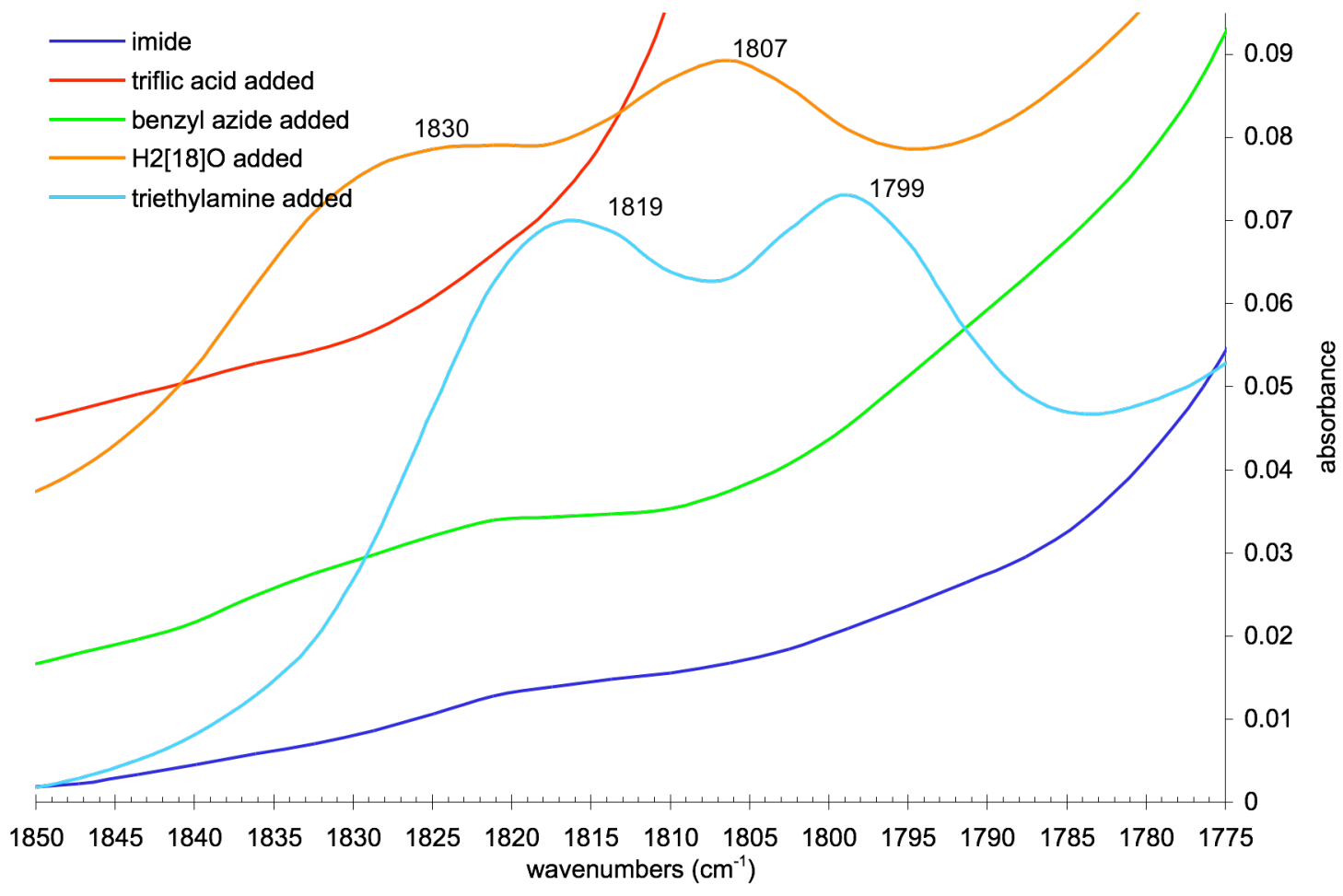

Thermal Conversion of Triazoline to Oxazolidine Dione Followed by $\mathrm{H}_{2}{ }^{18} \mathrm{O} 1850-1650 \mathrm{~cm}^{-1}$

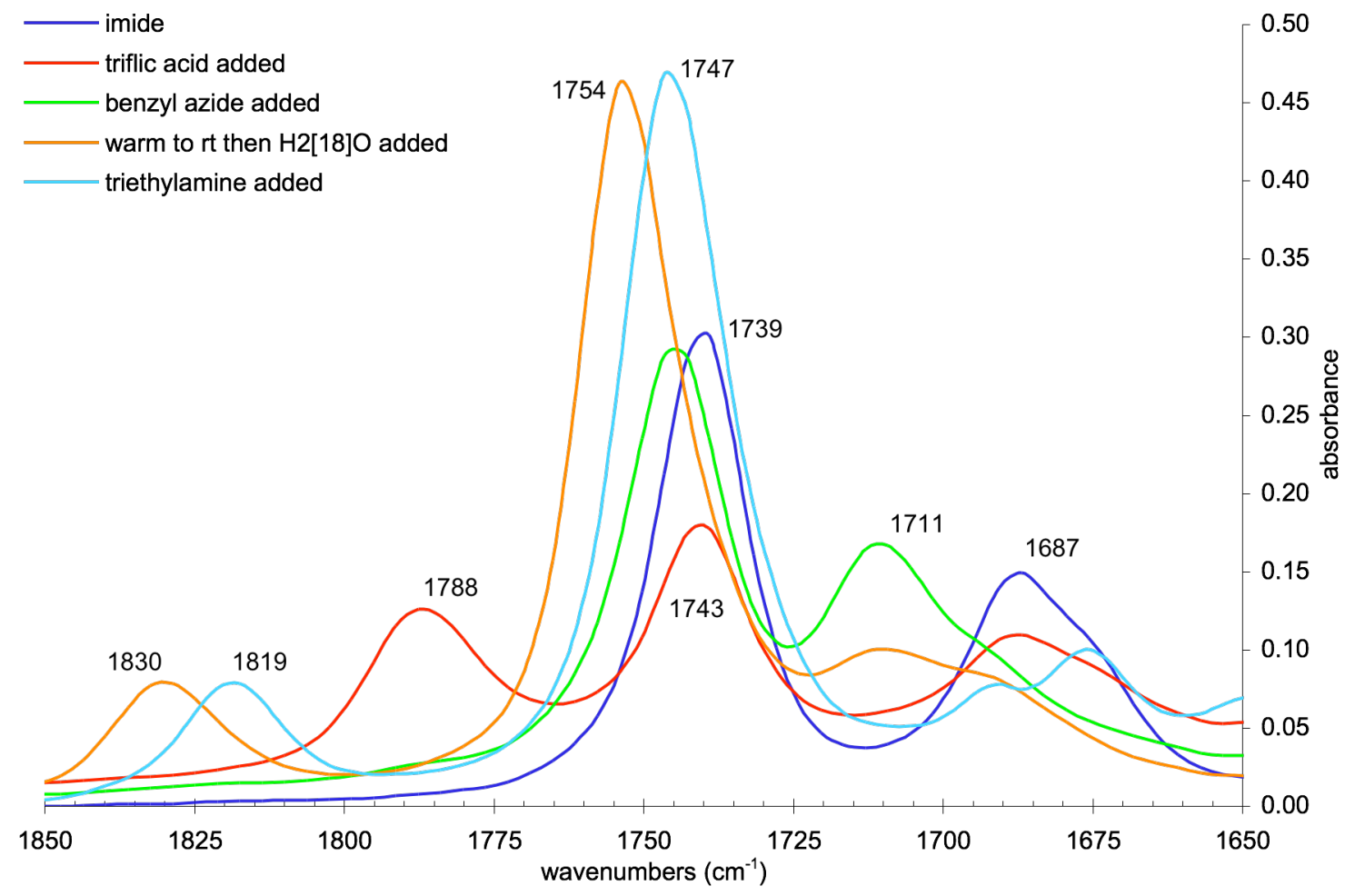

